\title{
EQUIPP-Report
}

\section{Initiative soll helfen, Europa rauchfrei zu machen}

— Jeder vierte erwachsene Bundesbürger raucht. Doch die Bundesregierung scheint wenig Interesse zu haben, das zu ändern: "Deutschland ist das tabakfreundlichste Land Europas", kritisiert Prof. Robert Loddenkemper, Berlin. Einen Grund hierfür sieht er in der einflussreichen Tabakindustrie, die 120 Mill. Euro pro Jahr in Werbung steckt und deren Lobbyisten eine schärfere Rauchpolitik verhindern. Die Politik gibt gerade mal 0,5 Mill. Euro jährlich für Maßnahmen gegen das Rauchen aus.

Die europaweite Initiative EQUIPP (Europe Quitting: Progress and Pathways) soll das ändern. Aus Interviews mit knapp 2500 Experten und je rund 20000 Rauchern und Nichtrauchern in 20 europä- ischen Ländern resultieren sieben zentrale Empfehlungen für Deutschland: Rauchverbot in der Öffentlichkeit, generelles Werbeverbot für Tabakwaren, Erhöhung der Tabaksteuer, Erstattung von Rauchentwöhnungsprogrammen und -therapien, Hausärzte als Zentrum der Rauchentwöhnung, Aufbau regionaler Rauchentwöhnungsnetzwerke, Rauchentwöhnung als Ausbildungsinhalt von Medizinstudenten und medizinischem Fachpersonal.

\section{"Tabaksucht ist eine Krankheit"}

Auch wenn die Tabakindustrie Rauchen als Lebensstilentscheidung darstellt, Tabaksucht ist eine Krankheit, betonte Prof. Bertrand Dautzenberg, Paris. Raucher zum Auf- hören zu bewegen, ist eine der effektivsten Vorbeugungsmaßnahmen gegen viele Krankheiten. Rauchern müssen mehr Angebote gemacht werden, die ihnen beim Rauchstopp helfen. Dazu zählt für Dautzenberg, dass Beratung und Therapie bezahlt werden: „Die Arzneimittel, die die Entwöhnung unterstützen, sind kosteneffektiv." Das britische National Institute for Clinical Excellence hält ein Pharmakon für kosteneffektiv, wenn es pro gewonnenem Lebensjahr nicht mehr 20000 Pfund kostet - die Rauchentwöhnungshilfen bringen es im Schnitt auf 800 bis 1000 Pfund.

- Manuela Arand

Quelle: EQIPP Launch Meeting, Berlin, März 2011 (Veranstalter: Pfizer)

\section{Chronische Obstipation}

\section{Enterokinetikum bringt den Darm Schwung}

Patienten mit chronischer Verstopfung leiden oft jahrelang unter Symptomen wie hartem Stuhl oder seltenen Darmentleerungen. Seit Anfang letzten Jahres steht mit Prucaloprid (Resolor ${ }^{\circledR}$ ) eine neue Therapieoption zur Verfügung, über die MMW mit Priv.-Doz. Dr. Jutta Keller, Hamburg sprach.

MMW: Für wen ist die Therapie mit Prucaloprid geeignet?

Keller: Die Therapie ist für Frauen mit chronischer Verstopfung gedacht, die auf die üblichen Medikamente nicht ausreichend ansprechen, sowie Patientinnen, die Abführmittel nicht vertragen.

MMW: Wie lange sollten Therapieversuche mit anderen Laxanzien dauern?

Keller: Das Vorgehen ist eher individuell. Es kommt z. B. auf die Schwere der Beschwerden an. Bei einem Patienten mit lästigen, aber milderen Beschwerden, hat man mehr Zeit zu versuchen, diese mit Laxanzien in den Griff zu bekommen. Wenn ein Patient trotz hoch wirksamer Laxanzien starke Beschwerden hat, wird er natürlich weniger Geduld haben und man wird früher zu Prucaloprid greifen.

MMW: Wie wirkt Prucaloprid? Keller: Prucaloprid wirkt gezielt an Serotonin(5-HT)-4-Rezeptoren in der Darmwand, die die Peristaltik im Darm anregen können. Der fast ausschließliche Wirkungsmechanismus von Laxanzien dagegen beruht darauf, dass sie für mehr flüssigen Darminhalt sorgen. Das kann sich indirekt auch auf die Bewegungsmuster auswirken, weil der Darm auf den Volumenreiz reagiert.

Studien zeigten, dass bei Patienten, die nicht auf die Therapie mit Laxanzien angesprachen, Prucaloprid die Häufigkeit von Stuhlgängen und das allgemeine Befinden deutlich bessern kann und dabei Placebo überlegen war. Auch die Lebensqualität wurde in einigen Studien erfasst und hat sich im Vergleich zu Placebo signifikant gebessert.

MMW: Welche unerwünschten Wirkungen können auftreten?

Keller: Prucaloprid führt relativ häufig gerade zu Beginn der Therapie zu Kopfschmerzen und zu einer Verschlechterung der Bauchbeschwerden. Meist hört dies nach ein bis zwei bei Priv.-Doz. Dr. med. Jutta Keller „Prucaloprid wirkt gezielt an $5-\mathrm{HT}_{4}{ }^{-}$ Rezeptoren, die die Peristaltik im Darm anregen."

Tagen Behandlung wieder auf. Es kam auch häufiger zu Durchfall als unter Placebo. Aber das ist weniger eine Neben-als ein Teil der Hauptwirkung. Ansonsten sind keine gravierenden Nebenwirkungen bekannt.

MMW: Lässt die Wirkung im Therapieverlauf nach?

Keller: Nach den bisher vorliegenden Daten über ein Jahr sieht es nicht so aus. Um diese Frage aber endgültig beantworten zu können, braucht man noch mehr Daten.

MMW: Wie hoch ist das Risiko einer QTZeitverlängerung?

Keller: Prucaloprid wirkt sehr gezielt am

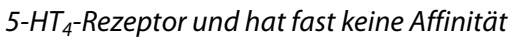
zu anderen Rezeptoren. In allen bisher vorliegenden Untersuchungen hat sich keine Verlängerung der QT-Zeit ergeben. Trotzdem wird bei der Kombination mit anderen Medikamenten, die die QT-Zeit verlängern können, Vorsicht empfohlen. 